\begin{tabular}{ccc}
\hline & $\begin{array}{c}\text { International Journal of Health Services } \\
\text { Research and Policy } \\
\text { www.dergipark.org.tr/ijhsrp }\end{array}$ \\
$\begin{array}{c}\text { INESEG } \\
\text { ENGINETIONAL } \\
\text { SCIENCE AND } \\
\text { EDUCATION GROUP }\end{array}$ & e-ISSN: $2602-3482$ & IJHSRP \\
\hline CaSE
\end{tabular}

Case report

\title{
LARYNGEAL TUBERCULOSIS - A NEGLECTED DISEASE THAT CAN MIMIC LARYNGEAL CARCINOMA
}

\author{
Jovan JAVORAC ${ }^{* 1,2}$ (1) Dejan ŽIVANOVIĆ ${ }^{2}$ (D) Aleksanra LOVRENSKI ${ }^{1,3}$ (i) \\ Ana MILENKOVIĆl ${ }^{1}$ Darinka KUKA VICA ${ }^{1}$ (D) Miroslav ILIĆ ${ }^{1,3}$
${ }^{1}$ Institute for Pulmonary Diseases of Vojvodina, Sremska Kamenica, Serbia
${ }^{2}$ College of Vocational Studies for the Education of Preschool Teachers and Sports Trainers, Dept. of Biomedical Sciences, Subotica, Serbia
${ }^{3}$ Faculty of Medicine, University of Novi Sad, Novi Sad, Serbia \\ * Corresponding author: jovanjavorac@vsovsu.rs
}

\begin{abstract}
Laryngeal tuberculosis is a form of extra-pulmonary tuberculosis that occurs in only $1 \%$ of all tuberculosis cases, with a mortality rate of less than $2 \%$. It can be a complication of pulmonary or some other form of extra-pulmonary tuberculosis (via bronchogenic, haematogenic, or lymphagenic spread of bacilli), or the larynx can be the only organ affected after the direct invasion of Mycobacterium tuberculosis. Clinical, laryngoscopic, and radiological findings of laryngeal tuberculosis tend to mimic laryngeal cancer, delaying the appropriate treatment. In this paper, we present a case of laryngeal and pulmonary tuberculosis, which was primarily diagnosed and almost treated as laryngeal carcinoma. An 84 years old Caucasian male presented with a 3-months history of hoarseness, odynophagia, dysphagia, cough with expectoration, and weight loss. After the laryngoscopy and biopsy of the laryngeal ulceration, a squamous cell carcinoma was suspected. However, chest $X$ ray and CT scan detected pulmonary infiltrates, while the positive sputum smear for acid-fast bacilli was obtained and the sputum cultures grew Mycobacterium tuberculosis. Detailed histopathological analysis of the biopsies was in accordance with tuberculosis, and no signs of timorous tissue were found. After the end of the proposed antituberculous treatment, the patient reported no symptoms of the diseases, laryngoscopic findings showed no signs of neoplastic tissue, and CT findings showed partial radiological regression. Distinguishing laryngeal carcinoma and tuberculosis can be very challenging, even after obtaining histopathological material. Nevertheless, biopsies should be taken from all suspicious lesions and at multiple sites, while Ziehl-Neelson staining of sputum and bioptic tissue is necessary. Otorhinolaryngologists should always consider tuberculosis in the differential diagnosis of laryngeal lesions, especially nowadays when the incidence of laryngeal tuberculosis is increasing in developing countries.
\end{abstract}

Keywords: tuberculosis, larynx, lung, carcinoma, misdiagnose. 


\section{Introduction}

Laryngeal tuberculosis is a form of extra-pulmonary tuberculosis that occurs in only $1 \%$ of all tuberculosis cases nowadays [1], with a mortality rate of less than 2\% [2]. However, in the first half of the 20th century, it was one of the most prominent forms of tuberculosis, affecting $35-83 \%$ of patients [3]. Due to the development of effective antituberculous drugs, improvement in living standards, and different public health interventions, the incidence of tuberculosis decreased significantly in the second part of the 20th century. Nowadays, due to the increasing prevalence of drug resistance, immunosuppressive conditions, poor living standards in some parts of the world, traveling to developing countries, and the spread of HIV infection, the incidence of tuberculosis, in general, is increasing [4]; therefore, it can be expected that the incidence of laryngeal tuberculosis could also rise in the upcoming years.

Laryngeal tuberculosis can be a complication of pulmonary tuberculosis when Mycobacterium tuberculosis has a bronchogenic spread from the lungs. Haematogenic or lymphogenic spread of bacilli from other, extra-pulmonary, organs are also possible. Solitary laryngeal involvement, when the larynx is affected via direct invasion of bacilli, is becoming more common nowadays [5]. Some studies suggest that laryngeal tuberculosis is found in around $40 \%$ of patients with no pulmonary manifestations [6].

Clinical, laryngoscopical, and radiological findings of laryngeal tuberculosis tend to mimic laryngeal carcinoma, delaying the appropriate treatment [7]. In this paper, we present a case of laryngeal and pulmonary tuberculosis which was primarily diagnosed and almost treated as laryngeal carcinoma.

\section{Materials and Methods}

\subsection{Case Description}

An 84 years old Caucasian male presented with a 3-months history of hoarseness, odynophagia, dysphagia, cough with expectoration, and loss of 10 kilograms of weight. He denied fever, chills, night sweats, or haemoptysis. The patient reported a history of previously treated pulmonary tuberculosis at the age of 18. He was a former tobacco consumer, did not use alcoholic beverages. After the completed fiberoptic laryngoscopy, an ulcerative lesion that almost completely destructed the region of the epiglottis was described. Biopsy of the epiglottis was performed two times in two different medical centers and the histopathological findings were suspected of squamous cell carcinoma of the mucosa in both of the cases. To investigate the dissemination of the disease, a chest X-ray (Fig. 1) and shortly after CT scans (Fig. 2A) were taken, with the radiological findings consistent to diffuse irregular lesions in the lung parenchyma, possibly as a part of pulmonary tuberculosis. The positive sputum smear for acidfast bacilli was obtained, while the sputum cultures, later on, grew Mycobacterium tuberculosis. Due to the possibility of coexistence of tuberculosis and cancer of the larynx, a more detailed analysis of paraffin moulds obtained from the biopsies was made; the histopathological features were consistent with tuberculosis, acid-fast bacilli were found, there was no neoplastic tissue in the examined material (Figure $3 \mathrm{a}$ and $3 b$ ). 


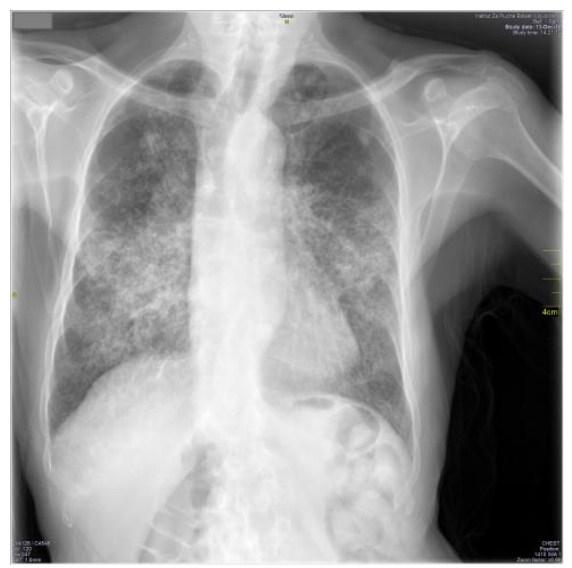

Figure 1. Initial chest X-ray

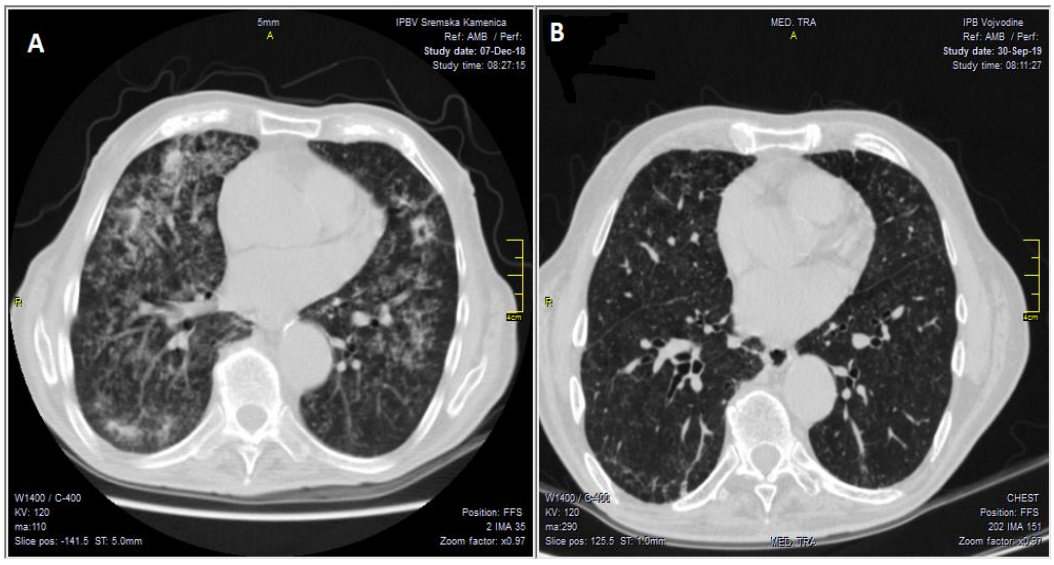

Figure 2. CT scans before (A) and after treatment (B)
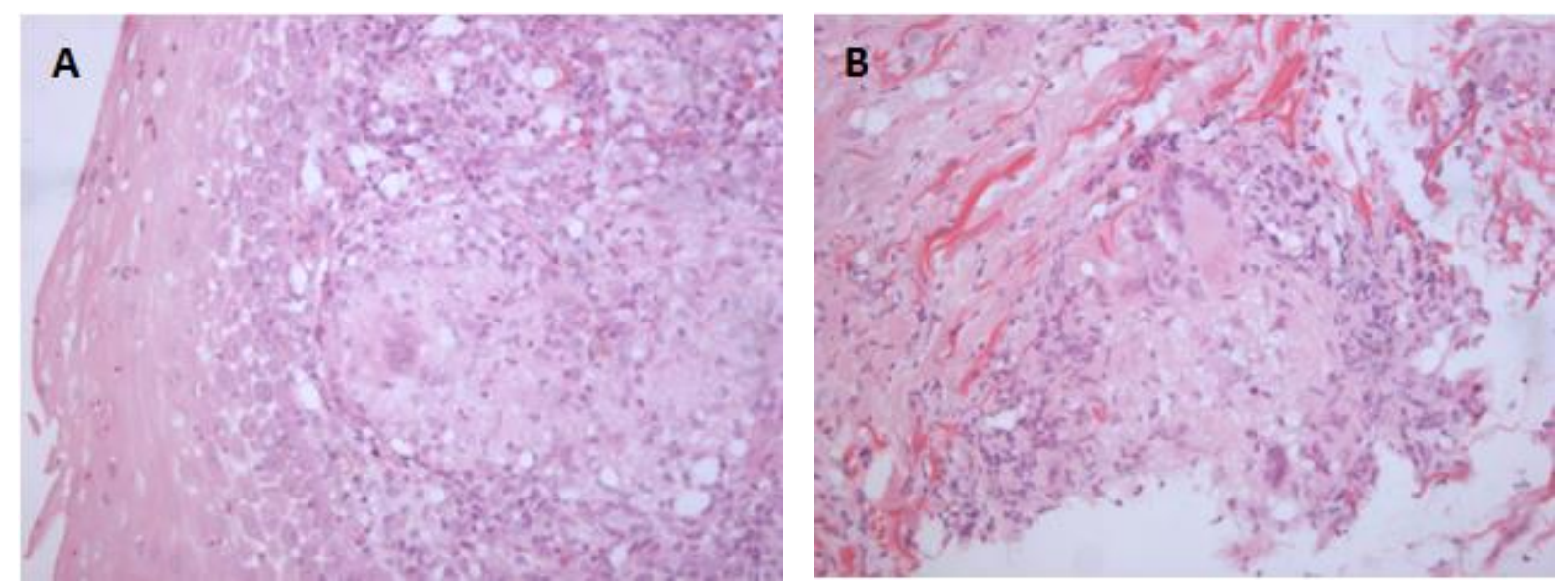

Figure 3. A squamous laryngeal epithelium on the surface, granulomas composed of epithelioid cells, lymphocytes, and giant Langhans cells are present in the mucosa, H\&E x 200 (A); a granuloma in the deeper layers of the wall made up of epithelioid cells, lymphocytes, and giant Langhans cells; an incipient necrosis is seen in the central part of the granuloma, H\&E x 200 (B).

Antituberculous therapy was administered (isoniazid $250 \mathrm{mg}$, rifampicin $450 \mathrm{mg}$, pyrazinamide $1200 \mathrm{mg}$, ethambutol $800 \mathrm{mg}$ ). Even though the patient was previously treated for tuberculosis, streptomycin was not introduced into therapy due to the patient's hearing loss, but an eight-month treatment regimen was implemented. During the course of treatment, he was regularly monitored by the pulmonologist. After 8 months of therapy, the patient was feeling completely healthy, reporting no symptoms of the disease. Laryngoscopally, no visible tumorous changes were detected, some parts of the epiglottis were missing though. CT scans of the chest (Fig. 2B) showed partial radiological regression of the previously described lung parenchyma alterations.

This study was approved by the Professional Board and the Ethics Committee of the Institute for Pulmonary Diseases of Vojvodina. Approval number and date: 112-I/3, 20.01.2021. 


\section{Discussion}

Even though tuberculosis is still the most common granulomatous disease of the larynx [1], clinicians usually do not consider it in the differential diagnosis when a lesion of the larynx is detected [8]. Initial diagnosis of laryngeal carcinoma is usually made in such cases because laryngeal tuberculosis and carcinoma share some important demographic, clinical, laryngoscopical, and radiological features. Laryngeal carcinoma occurs 40 times more often than tuberculosis, as well [9].

Laryngeal tuberculosis nowadays usually affects those between 40 and 60 years, which is different from the past, when those between 20 and 39 were usually affected. Also, it is notable that males are at a greater risk of infection, while in the past no gender predominance was present $[5,10,11]$. Similar demographic characteristics can be found in patients with laryngeal carcinoma. Risk factors, such as cigarette smoking, alcohol abuse, poor nutrition, workplace exposures, can be mutual as well, all leading to diagnostic difficulties [7]. Underlying conditions, such as HIV infection or diabetes, should always be considered. In some studies, it was estimated that HIV infection was present in $48 \%$ of patients with head and neck tuberculosis, and $25 \%$ of patients with laryngeal tuberculosis [9].

Laryngeal tuberculosis and carcinoma can have similar clinical features, such as hoarseness, odynophagia, dysphagia, cough, stridor, hemoptysis, and cervical lymphadenopathy [12]. Constitutional symptoms, such as loss of weight, fever, malaise, night sweats, can be also presented in both cases. The main complaint of patients with laryngeal tuberculosis is hoarseness (70-97\% of cases), followed by odynophagia in around $40 \%$ of cases [5,11], unlike in the past, when patients usually had dyspnea or pulmonary and other constitutional symptoms. Odynophagia is not common in laryngeal carcinoma, and this could be an important clue for distinguishing two similar diseases [13].

Laryngoscopic findings of tuberculosis may be divided into four different types: granulomatous, ulcerative, polypoid, and nonspecific lesions (such as diffuse oedema and inflammation) [4,5]. All of these forms can be seen in laryngeal carcinoma, especially hypertrophic, exophytic and polypoid lesions, aggravating the distinction between two laryngeal diseases. When considering the localization of the lesions, posterior parts of the larynx were the most common site of infection in the past (when the lung involvement and bronchogenic spread of bacilli were often), but nowadays, the anterior part of the larynx is more affected [14], with vocal cords being affected predominantly [3].

In the initially suspected diagnosis of carcinoma, laryngeal tuberculosis is generally not considered until the chest X-ray or CT scan is done. In patients with tuberculosis, the chest X-ray is usually abnormal and often appears with cavitations at the apical parts of the lung together with bilateral nodular infiltrates [15]. In a systematic review from 2014, lung involvement on chest X-ray was found in $86 \%$ of confirmed cases of laryngeal tuberculosis [1]. In a study of 60 cases of laryngeal TB, only $20 \%$ had normal chest radiographs [4]. All of this suggests that chest radiography should be an inevitable diagnostic procedure when examining laryngeal lesions. Radiological imaging in solitary laryngeal tuberculosis is non-specific. CT scan and MR imaging of the neck usually show diffuse bilateral thickening or a mass in the vocal cords, epiglottis, or paralaryngeal tissue in the acute phase [16]. The typical finding in the chronic phase is a localized lesion mimicking a mass [17].

Even though the finding of acid-fast bacilli in sputum smear is suggestive of tuberculosis, the positive rate of sputum smear was only around $43 \%$ in a study by Wang et al [9]. In other studies, the positive rate of sputum culture was higher (around $85 \%$ ), but it takes several weeks to obtain the result [18]. 
The final diagnostic tool to distinguish carcinoma and tuberculosis of larynx should be the histopathological analysis of the material obtained after the biopsy of the laryngeal lesion. The findings of granulomatous inflammation, caseating granuloma, or acid-fast bacilli confirm the diagnosis of tuberculosis [10]. However, the presence of pseudoepitheliomatous hyperplasia that mimics squamous cell carcinoma can make the diagnosis difficult $[2,19]$. The physician must be aware that tuberculosis and carcinoma of the larynx can co-exist, even though that happens seldom. A patient with carcinoma may have deficient cellular immunity, leading to the development of tuberculosis [11].

Once the diagnosis of laryngeal tuberculosis is confirmed, immediate treatment should be implemented, using antituberculous drugs, administered for a minimum of six months [20], even though some researchers suggest that the treatment should be extended to one year [21]. During the course of treatment, it is important to obtain the sensitivity results and correct the therapy if necessary. Most lesions resolve over two months, while the patient is considered to be cured after the end of the implemented treatment protocol and after the confirmation of inactive disease through sputum microbiological controls, chest X-ray, and laryngoscopy [22]. Even though the treatment is usually effective, untreated or incorrectly treated laryngeal tuberculosis can lead to laryngeal stenosis and/or cricoarytenoid fixation [23], requiring surgical interventions.

The typical situation of misdiagnosis was described in this case report. Because of the clinical symptoms, age of the patient, history of previously cigarette smoking, and finding of ulceration of epiglottis spreading into the surrounding areas during fiberoptic laryngoscopy, the preliminary diagnosis was carcinoma. However, the most intriguing fact was that two biopsies were performed and both indicated squamous cell carcinoma, but no Ziehl-Neelson staining of biopsies or sputum was performed. Only after applying this method, usage of imaging methods, and detailed examination of biopsied tissue, the diagnosis of tuberculosis was confirmed. Thus, it is not surprising that some authors suggest that in an endemic area for tuberculosis, samples of laryngeal lesions should be reviewed by two pathologists [10]. The diagnosis of carcinoma was excluded for certain after the 8 months of therapy for tuberculosis when the patient had no clinical, laryngoscopical, or radiological signs of carcinoma.

\section{Conclusions}

Distinguishing laryngeal carcinoma and tuberculosis can be very challenging. The diagnostic algorithm should start with thorough history taking, to identify signs and symptoms of the disease, risk factors, and eventual contact with a person who had similar complaints. The diagnostic approach should also include chest radiographs and examination of sputum for acid-fast bacilli (smears and cultures) to exclude or confirm concomitant pulmonary tuberculosis. If those additional exams are not indicating pulmonary tuberculosis, laryngoscopy should be performed and biopsies of the laryngeal lesions should be taken from all suspicious lesions and at multiple sites with Ziehl-Neelson staining of the obtained material.

Otorhinolaryngologist should always consider tuberculosis when evaluating laryngeal lesions, especially nowadays when the incidence of laryngeal tuberculosis is increasing in developing countries. The early distinction of these two diseases does not only affect the treatment but is also essential in disease transmission control and decreasing the risk of sequelae. 
Ethical considerations: This study was approved by the Professional Board and the Ethics Committee of the Institute for Pulmonary Diseases of Vojvodina. Approval number and date: 112-I/3, 20.01.2021. Informed consent has been obtained from the patient whose case was described in this study.

Conflict of interest: The authors declare that they have no conflict of interest nor any financial support in the preparation of this study.

\section{References}

[1] Benwill, J. L., \& Sarria, J. C., "Laryngeal tuberculosis in the United States of America: a forgotten disease", Scandinavian Journal of infectious diseases, 46 (4), 241-249, 2014. https://doi.org/10.3109/00365548.2013.877157

[2] Uslu, C., Oysu, C., Uklumen, B., "Tuberculosis of the epiglottis: a case report", European archives of oto-rhino-laryngology, 265 (5), 599-601, 2008. https://doi.org/10.1007/s00405-007$\underline{0492-9}$

[3] Rizzo, P.B., Da Mosto, M.C., Clari, M., Scotton, P.G., Vaglia, A., Marchiori, C., "Laryngeal tuberculosis: an often forgotten diagnosis", International Journal of infectious diseases, 7 (2), pp.129-131, 2003. https://doi.org/10.1016/S1201-9712(03)90008-7

[4] Paulauskienė, I., \& Mickevičienė, V., "Dysphonia-the single symptom of rifampicin-resistant laryngeal tuberculosis", Open Medicine, 11 (1), 63-67, 2016. https://doi.org/10.1515/med-2016$\underline{0013}$

[5] Lim, J.Y., Kim, K.M., Choi, E.C., Kim, Y.H., Kim, H.S., Choi, H.S., “Current clinical propensity of laryngeal tuberculosis: review of 60 cases", European Archives of Oto-RhinoLaryngology and Head \& Neck, 263 (9), 838-842, 2006. https://doi.org/10.1007/s00405-006$\underline{0063-5}$

[6] Shin, J.E., Nam, S.Y., Yoo, S.J., Kim, S.Y., "Changing trends in clinical manifestations of laryngeal tuberculosis", Laryngoscope, 110, 1950-1953, 2000. https://doi.org/10.1097/00005537-200011000-00034

[7] Lou, Z.C., \& Li, X., "Leukoplakia or LPR: The Misdiagnosis of Laryngeal Tuberculosis", Ear, Nose \& Throat Journal, pp.0145561319891264, 2019. https://doi.org/10.1177/0145561319891264

[8] González-Martín, J., García-García, J.M., Anibarro, L., Vidal, R., Esteban, J., Blanquer, R., Moreno, S., Ruiz-Manzano, J., "Consensus document on the diagnosis, treatment and prevention of tuberculosis", Archivos de Bronconeumología (English Edition), 46 (5), 255-274, 2010. https://doi.org/10.1016/S1579-2129(10)70061-6

[9] Wang, C.C., Lin, C.C., Wang, C.P., Liu, S.A., Jiang, R.S., "Laryngeal tuberculosis: a review of 26 cases", Otolaryngology-Head and Neck Surgery, 137 (4), 582-588, 2007. https://doi.org/10.1016/j.otohns.2007.04.002

[10] Hasibi, M., Yazdani, N., Asadollahi, M., Sharafi, M., Manshadi, S.A.D., "Clinical features of laryngeal tuberculosis in Iran”, Acta Medica Iranica, 51 (9), 638-641, 2013. 
[11] Ling, L., Zhou, S.H., Wang, S.Q., "Changing trends in the clinical features of laryngeal tuberculosis: a report of 19 cases", International Journal of Infectious Diseases, 14(3), e230e235, 2010. https://doi.org/10.1016/j.ijid.2009.05.002

[12] Smulders, Y.E., De Bondt, B.J., Lacko, M., Hodge, J.A., Kross, K.W, "Laryngeal tuberculosis presenting as a supraglottic carcinoma: a case report and review of the literature", Journal of Medical Case Reports, 3 (1),9288, 2009. https://doi.org/10.1186/1752-1947-3-9288

[13] Lodha, J.V., Sharma, A., Virmani, N., Bihani, A., Dabholkar, J.P., "Secondary laryngeal tuberculosis Revisited", Lung India, 32, 462-464, 2015. https://doi.org/10.4103/09702113.164163

[14] Nalini B., \& Vinayak S., "Tuberculosis in ear, nose, and throat practice: its presentation and diagnosis", American Journal of otolaryngology, 27 (1), 39-45, 2006.

https://doi.org/10.1016/j.amjoto.2005.07.005

[15] Kurokawa, M., Nibu, K.I., Ichimura, K.I., Nishino, H, "Laryngeal tuberculosis: A report of 17 cases", Auris Nasus Larynx, 42 (4), 305-310, 2017. http://dx.doi.org/10.1016/j.anl.2015.02.012

[16] Cengiz, A., Göksel, S., Başal, Y., Gülen, Ş.T., Döğer, F., Yürekli, Y., “Laryngeal tuberculosis mimicking laryngeal carcinoma on 18F-FDG PET/CT Imaging", Molecular imaging and radionuclide therapy, 27(2), 81-83, 2018. https://doi.org/10.4274/mirt.44366

[17] Moon, W.K., Han, M.H., Chang, K.H., Kim, H.J., Im, J.G., Yeon, K.M., Han, M.C., "Laryngeal tuberculosis: CT findings," American Journal of roentgenology, 166(2),445-449, 1996. https://doi.org/10.2214/ajr.166.2.8553964

[18] Gambhir, S., Ravina, M., Rangan, K., Dixit, M., Barai, S., Bomanji, J., ’Imaging in extrapulmonary tuberculosis", International Journal of Infectious Diseases, .56, 237-247, 2017. https://doi.org/10.1038/s41426-017-0008-7

[19] Yencha, M.W., Linfesty, R., Blackmon, A., "Laryngeal tuberculosis", American Journal of otolaryngology, .21 (2), 122-126, 2000. https://doi.org/10.1016/S0196-0709(00)85010-3

[20] Qian, X., Albers, A.E., Nguyen, D.T., Dong, Y., Zhang, Y., Schreiber, F., Sinikovic, B., Bi, X., Graviss, E.A., "Head and neck tuberculosis: literature review and meta-analysis", Tuberculosis, 116, S78-S88, 2019. https://doi.org/10.1016/j.tube.2019.04.014

[21] Reis, J.G.C., Reis, C.S.M., Costa, D.C.S.D., Lucena, M.M., Schubach, A.D.O., Oliveira, R.D.V.C., Rolla, V.C., Conceição-Silva, F., Valete-Rosalino, C.M., "Factors associated with clinical and topographical features of laryngeal tuberculosis", PLoS One, 11(4),e0153450, 2016. https://doi.org/10.1371/journal.pone.0153450

[22] Alegre, J.L.H., Gutierrez, A.T., Prado, E.M., Marcelo, J.H., "Laryngeal Tuberculosis in Pregnant Women: A Case Report and Review of the Literature", Cureus, 10(11), e3545, 2018. https://doi.org/10.7759/cureus.3545

[23] Edizer, D.T., Karaman, E., Mercan, H., Alimoglu, Y., Esen, T. Cansiz, H., "Primary tuberculosis involving epiglottis: a rare case report", Dysphagia, 25 (3), 258-260, 2010. https://doi.org/10.1007/s00455-009-9256-6 